\title{
The Effect of Group Size and Communication Modes in CSCW Environments
}

\author{
Masood Masoodian \\ Department of Computer Science \\ University of Waikato, Hamilton, New Zealand \\ masood@cs.waikato.ac.nz \\ Mark Apperley \\ Department of Computer Science \\ University of Waikato, Hamilton, New Zealand \\ m.apperley@cs.waikato.ac.nz
}

\begin{abstract}
An earlier study [7] has examined the impact of different human-to-human communication modes on computer supported work involving groups of two people. Based on the findings of this study an experiment was designed to explore the relative impact of face-to-face and audio-only communication modes in supporting shared-workspace interaction between groups of three people. This paper describes the experiment, and examines its findings with the aim of establishing the relationship between the effectiveness of the various communication modes and the group size.

Keywords: computer supported cooperative work (CSCW), group size, group communication, group work, communication mode, problem-solving, shared workspace.
\end{abstract}

\section{Introduction}

Over the last two decades the importance of group work, particularly computer supported group work, has been realised by both researchers and commercial organisations. This realisation has in turn led to an increased interest in the study of various aspects of group work.

Although there are a large number of factors that affect the process of group work, three of these factors could be considered more important than others. Williams [22] identifies these factors to be the communication modes available, the nature of the task involved, and the size of the group.

There is a growing volume of literature reporting studies whose purpose is to compare various forms of constrained human-to-human communication $[13,17,22$, 19, 12, 16]. These studies have focused on different configurations of text-based, audio, video and face-to- face* meeting environments with the aim of establishing their effectiveness in supporting different types of collaborative group work.

There are also studies which have examined a variety of group tasks [2, 15, 13]. McGrath [9] has given a classification of these tasks together with a list of studies which have utilised them. He identified eight different group task types, which he then categorised into two groups of cooperative and conflict type. The cooperative task category, which includes planning, creative, intellective (problem-solving), and psycho-motor types, represents the tasks that have to be "solved". The conflict task types (decision-making, cognitive conflict, mixedmotive, and competitive) on the other hand, are the tasks that have to be "resolved". This may involve resolving conflict of view-points or conflict of interests between the group members. It should be noted that McGrath's task classification is not the only one available; it is however derivative of a number of others and is by far the most commonly used task type classification.

A review of the current literature on group work and human-to-human communication would show that most of the published research in this area has considered the two major variables of media of communication and communication task, while ignoring the third important factor of group size. In fact the majority of these studies were carried out with groups of just two people [2, 12, 14, 15, 18]. However as Williams [22] suggests, since group processes are more complex in larger groups, it is reasonable to predict that the effects of media differences are more likely to appear as the group size increases.

Although in the past few years there have been a number of studies that have concentrated on larger groups of three $[10,13]$ or four $[3,16]$, there are few that have focused on the group size as the main factor under investigation. One such study has been carried out by Valacich et al. [20]. This study, which is one of a series, investigates the effects of group size on the process of

\footnotetext{
* The term face-to-face is commonly used in the CSCW literature to describe situations where participants are co-located and in view of one another.
} 
idea-generation in computer-based and non-computerbased work environments. It was found that large groups using a computer-based idea-generation system outperformed groups that did not use such system. Groups working in a computer-base environment also outperformed nominal groups (equivalent numbers of individuals working alone).

This paper reports on an experiment designed to study the relative effectiveness of audio and face-to-face communication modes in a cooperative shared-workspace problem-solving situation involving groups of three people. The experiment extends and complements an earlier study which examined audio, video, and face-toface communication modes in supporting two person groups.

It should be noted that unlike many other similar studies, the work described in this paper focuses mainly on variation in group size as an important aspect of computer supported collaborative work.

\section{Background}

A recent study $[7,5,6]$ investigated the effects of four human-to-human communication modes in a sharedworkspace computer supported cooperative work environment. These modes were:

- face-to-face meetings

- $\quad$ remote meetings supported by an audio link

- $\quad$ remote meetings supported by a full motion video link

- $\quad$ remote meetings supported by a slow motion (five frames per second) video link

Twelve groups of two people worked in each of these environments on a set of four computer-based jigsaw puzzles, each in a different sequence. Two sets of data were collected; (i) through videotaping the subjects' upper body area and their workspace during the sessions, and (ii) through using a set of three questionnaires. These data were analysed using a number of subjective and objective methods.

The results of this study [7] clearly showed that in a computer-supported shared-workspace problem-solving situation, audio communication is very important. This confirmed the results of Chapanis and Ochsman's work [2, 12] which had examined the differences between various communication modes in supporting collaborative noncomputer-based problem-solving work. Sellen [16] reported similar results from an experiment which compared three different types of video communication with the normal face-to-face and audio-based communication modes, using conflictive type tasks.

This study [7] also indicated that there was no significant difference in the style of interaction between subjects, or the time taken to solve problems, between any of the audio-based, video-based, or face-to-face communication modes. The study indicated that the only difference between these communication modes was reflected in the users' perception of the media [5]. Users felt that video added value, and video-based or face-toface communication seemed more "natural". This result was also in agreement with the findings of other related studies [3, 13].

Since this empirical study investigated variations to the medium of communication, the other major factors that could be considered for future extensions to this work were the effects of changing the task type and the group size.

As was discussed in Masoodian et al. [7], for an experiment of this type, which was designed to study the effects of different communication media in a computersupported cooperative work environment, it is only reasonable to use cooperative tasks. Also, of the four tasks that McGrath [9] classifies as cooperative, only intellective (problem-solving), planning, or creativity type tasks are suitable for a computer-based work environment (the psycho-motor type tasks are physical tasks which can not be performed on computers). Therefore the variation that can be made to the task type for this kind of study is limited. There is also a certain amount of evidence from other work $[17,22,9]$ that the effects of changing the type of cooperative task would have very little effect on the group communication process.

Thus there was incentive to first consider changing the group size and studying its effects on the group communication process. The remainder of this paper describes an experiment which extends the earlier work mentioned above. The group size for this experiment was changed from two to three people per group. The task type, however, remained the same. In fact to make it possible to compare the results of this experiment with the previous one [7], the same set of four jigsaw puzzles were used. In the previous study jigsaw puzzles proved to be very successful and they encouraged the subjects to work to the best of their ability. Each puzzle had a number of tricky parts which required the subjects to cooperate and communicate with one another; without this cooperation and communication the puzzles would have been difficult to complete.

\section{Experiment}

Two different modes of meeting were selected for this study. These were: face-to-face meetings, and physically separated meetings supported by an audio link. Although video is usually considered as a communication mode that falls between audio and face-to-face communication modes [7], it was not used in this experiment. There were several reasons for this. First, logic would suggest that in terms of effectiveness, video would come between audio and face-to-face [7] yet the earlier experiment had detected no difference in performance between all three. Second, recent work by Olson et al. [13] shows that, in a similar situation, any observable difference between the audio, video, and face-to-face modes existed only between the audio and the face-to-face communication modes. Third, the physical problems of providing views of 
multiple participants are more difficult to overcome. However, it was decided that if the results did show any difference in this respect then a further experiment would be carried out to include the video communication mode as well.

Both of the communication modes utilised a computerbased shared-workspace system, provided by the Aspects $^{\mathrm{TM}}$ [4] conferencing software and three linked Macintosh ${ }^{\mathrm{TM}}$ computers. Aspects software allowed the subjects to work interactively on shared drawing documents. A shared workspace was used because it is an important element of synchronous computer supported cooperative work, and other research [21] has demonstrated its effectiveness in supporting collaborative group work.

It is appreciated that Aspects, like other software of this type has a number of shortcomings in such areas as the interface, responsiveness, and floor control. However, because it was used in both of the two settings, the impact of these shortcomings on the communication between the subjects was reduced.

\subsection{Face-to-face meeting environment}

As Figure 1 shows, in this environment the subjects sat in front of their computers in a triangular setting facing each other. The subjects were able to see each other's upper-body areas.

To keep the recorded sound quality uniform across the two environments, even in the face-to-face mode the subjects communicated through a headset sound system. This in fact also eliminated any differences that might have existed between the sound quality in the face-to-face and voice-only communication modes. Although this made the communication rather different from the usual face-to-face communication, the subjects had no difficulty in adopting to it and they felt that the interaction remained remarkably natural.

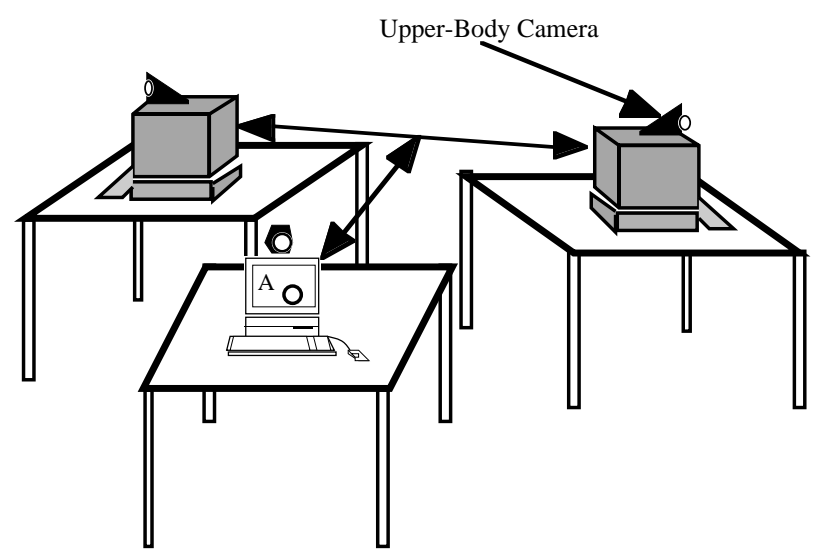

Figure 1: Face-to-face meeting environment

Three video cameras were present in the meeting room. These cameras were placed on top of the computer monitors and they recorded the upper-body area of each of the subjects. There was also a fourth computer in the control room which was linked to the computers of the three subjects and showed a view of their shared workspace. The video signals from the three cameras and the control room computer, plus the spoken dialogue between the subjects, were recorded on a single video tape using a four-quadrant video mixer. This was done so that it would be possible during the analysis to simultaneously observe the view of the upper-body area of each of the subjects and their shared workspace. Figure 2 shows a single frame from one of the video tapes.

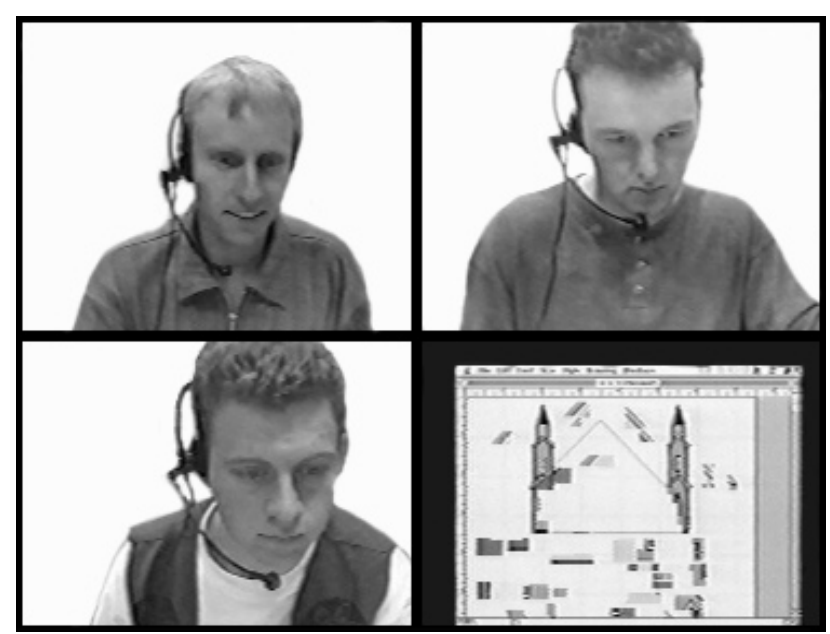

Figure 2: A single frame of a recorded session showing the three participants and the shared workspace which they each are viewing

\subsection{Voice-only meeting environment}

The subjects in the second environment (Figure 3) worked on three linked computers located in separate rooms. Each subject was provided with an audio link to the others via a headset. Once again, there was a video camera placed on the computer monitor in each room which captured the images of the upper-body area of the subject working in that room. As with the face-to-face situation, the images from the three cameras, the control room computer (showing the shared workspace), and the audio conversations between the subjects, were recorded on a single video tape for analysis purposes.

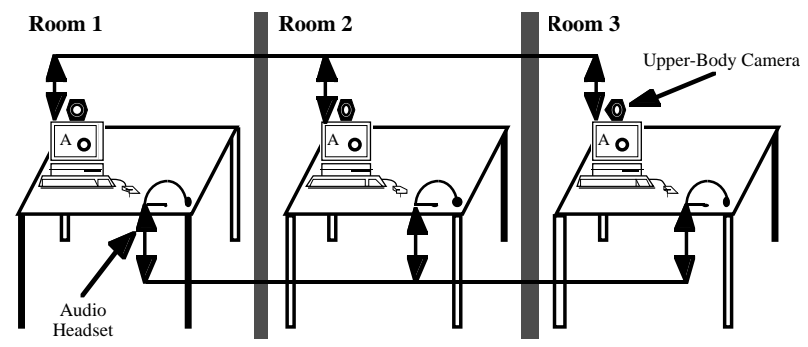

Figure 3: Voice-only meeting environment 


\section{Design}

McCarthy and Monk [8] have identified three experimental designs for assigning subjects to groups and groups to different experimental conditions. These are: between subjects/between groups, within subjects/within groups, and within subjects/between groups. In the between subjects/between groups design each subjects takes part in only one group and each group works in only one of the experimental settings. In the within subjects/within groups design however, each subject is assigned to only one group but each group works in all of the environments. Within subjects/between groups is the most complicated design. In this design the subjects work in all the experimental conditions but they are put into new groups for each condition.

For this experiment, within subjects/within groups design was chosen. This design uses fewer subjects and allows them to be able to compare the environments. This design is also more sensitive because it makes it possible to separate the individual differences from the error variance [8]. However, with this design it is important to make sure that each ordering of the environments is experienced by the same number of groups. Four groups of three people were used for this experiment. All the subjects were native-English-speaking males who volunteered to participate. The subjects were all computer science students and they all had good computer skills. Four of the subjects were postgraduate and eight of them were third or fourth-year undergraduate students. The subjects were assigned to the groups randomly.

Table 1 shows the ordering of the environments used in this experiment. Each ordering was replicated twice. Numbers 1 to 4 (in bold style) show the sequence in which each group worked in the different environments. Each of these numbers also identifies a task on which the given group worked in that environment. Each group performed two tasks in each environment so that the number of samples taken from the environments could be increased while the number of the subjects remained the same. Therefore overall eight samples were taken from each of the environments.

\begin{tabular}{|c|c|c|c|c|}
\hline Group Mode & \multicolumn{2}{|c|}{ Voice-Only } & \multicolumn{2}{c|}{ Face-to-Face } \\
\hline 1 & $\mathbf{1}$ & $\mathbf{2}$ & $\mathbf{3}$ & $\mathbf{4}$ \\
\hline 2 & $\mathbf{1}$ & $\mathbf{2}$ & $\mathbf{3}$ & $\mathbf{4}$ \\
\hline 3 & $\mathbf{4}$ & $\mathbf{3}$ & $\mathbf{2}$ & $\mathbf{1}$ \\
\hline 4 & $\mathbf{4}$ & $\mathbf{3}$ & $\mathbf{2}$ & $\mathbf{1}$ \\
\hline
\end{tabular}

Table 1: Ordering of the environments

\section{Data}

During this experiment two sets of data were collected. The first set was obtained from three different questionnaires. At the beginning of sessions one and three, when the subjects were introduced to a new environment, they were asked to fill out a questionnaire that recorded their expectations of the environment in which they were about to work (questions 1.1 to $1.5 \mathrm{D}$ in Table 2).

At the end of the second and fourth sessions, when the subjects had finished working in an environment, they were given another questionnaire which recorded their reactions to that environment (questions 2.1 to 2.9 in Table 2). There was also a third questionnaire which was used at the end of the experiment (end of session four) to get the subjects' ranking of the two environments (questions 3.1 to 3.5 in Table 2).

The second type of collected data was the set of video tapes which contained the recorded images of the upperbody area of the three subjects in each group and the image of their shared workspace, plus the audio conversation between the subjects. The video and audio signals were passed through a four channel video mixer and an audio mixer and were recorded on a single video tape. This was done so that the audio and video signals were synchronised for later analysis.

\section{Results}

The collected data from this experiment were analysed in terms of the style of the conversation between the subjects, the time taken to complete the tasks in each of the environments, and the subjects' response to the questionnaires.

The next two sections describe the results of these analyses in detail.

\subsection{Interaction style and session duration}

The analysis method for the style of conversation [14, 15] is very similar to the one used in the previous study [7]. However, since there were three subjects in each group who communicated with one another (rather than the previous dyadic conversation), the method had to be changed slightly to include a number of additional variables. The following factors were measured: total speech duration, number of utterances, total duration of simultaneous speech involving two people, total duration of simultaneous speech involving three people, number of simultaneous speech utterances involving two people, number of simultaneous speech utterances involving three people, total duration of mutual silence, number of floor control changes, and the number of unsuccessful attempts to gain the floor control.

No predictions were made about the value of these variables in this experiment and they were compared only across the two different environments. 


\begin{tabular}{|c|c|}
\hline Question & Answer \\
\hline $\begin{array}{l}\text { 1.1- How satisfied do you think you will be with this communication mode? } \\
\text { 1.2- How easy do think it will be to communicate in this environment? } \\
\text { 1.3- How efficiently do you think you can work in this environment? } \\
\text { 1.4- How enjoyable do think it will be to work in this environment? } \\
\text { 1.5- Indicate your expectations about how it would be to work in this environment: } \\
\text { 1.5A } \\
1.5 \mathrm{~B} \\
1.5 \mathrm{C} \\
1.5 \mathrm{D}\end{array}$ & $\begin{array}{l}\text { (1) Very Dissatisfied to (7) Very Satisfied } \\
\text { (1) Very Difficult to (7) Very Easy } \\
\text { (1) Not Very Efficiently to (7) Very Efficiently } \\
\text { (1) Not Very Enjoyable to (7) Very Enjoyable } \\
\text { (1) Very Impersonal to (7) Very Friendly } \\
\text { (1) Very Frustrating to (7) Not Very Frustrating } \\
\text { (1) Very Time Wasting to (7) Very Time Saving } \\
\text { (1) Very Unproductive to (7) Very Productive }\end{array}$ \\
\hline $\begin{array}{l}\text { 2.1- How satisfied are you with this communication mode? } \\
\text { 2.2- How much did you enjoy working on the last two tasks? } \\
\text { 2.3- How efficient was the group work over the last two sessions? } \\
\text { 2.1- How easy was it to control the communication in this environment? } \\
\text { 2.5- How satisfied are you with the group work you did in the last two sessions? } \\
\text { 2.6- How easy was it to understand your partners' ideas in this environment? } \\
\text { 2.7- How easy was it to get your own ideas across in this environment? } \\
\text { 2.8- How much did the other participants contribute to the group work in the last two sessions? } \\
\text { 2.9- How much did you contribute to the group work in the last two sessions? }\end{array}$ & $\begin{array}{l}\text { (1) Very Dissatisfied to (7) Very Satisfied } \\
\text { (1) Very Little to (7) Very Much } \\
\text { (1) Not Very Efficient to (7) Very Efficient } \\
\text { (1) Very Difficult to (7) Very Easy } \\
\text { (1) Very Dissatisfied to (7) Very Satisfied } \\
\text { (1) Very Difficult to (7) Very Easy } \\
\text { (1) Very Difficult to (7) Very Easy } \\
\text { (1) Very Little to (7) Very Much } \\
\text { (1) Very Little to (7) Very Much }\end{array}$ \\
\hline $\begin{array}{l}\text { 3.1- How enjoyable was it to work in the two environments? } \\
\text { 3.2- How efficiently did you work in the two environments? } \\
\text { 3.3- How easy was it to communicate in the two environment? } \\
\text { 3.4- How easy was it to work in the two environments? } \\
\text { 3.5- How would you rank the two environments overall in terms of your own preference? }\end{array}$ & $\begin{array}{l}\text { (1) Most Enjoyable to (2) Least Enjoyable } \\
\text { (1) Most Efficient to (2) Least Efficient } \\
\text { (1) Easiest to (2) Hardest } \\
\text { (1) Easiest to (2) Hardest } \\
\text { (1) Best to (2) Worst }\end{array}$ \\
\hline
\end{tabular}

Table 2: A summary of the three questionnaires

The variables were scored directly from the recorded video tapes of the sessions by an observer, using a computer program specifically written for this purpose. The recorded video tapes of the sessions were viewed and depending on whether a subject was talking or not at a given time, a key on the computer keyboard assigned to that subject was pressed or released. Based on these input values the program generated a series of on/off patterns for each of the group members through the entire session. The same observer viewed all the video tapes session by session (rather than group by group, i.e. first sessions for all the groups were viewed before the second sessions and so on) so that the accuracy of the logging remained similar between the groups

The conversation patterns generated by the first program were then passed through another custom-made program which analysed them and produced a value for each of the measured variables. This program was based on the approach used by Argyle and Cook [1] for measuring gaze and speech patterns. However, the method had to be extended considerably to be used for analysing 3 -way conversations. The final method is very similar to the one used by Sellen [16].

The session duration was also measured from the video tapes. Only one of the sixteen sessions did not produced a completed jigsaw puzzle. To avoid having a missing value for this session in the analysis process, it was assumed that the session would have taken a long time to complete. A ratio of maximum allowed time over actual session duration was used to compare the time taken to complete the tasks in two environments so that the value used for the incomplete session would be zero. The maximum allowed time was 40 minutes for the third task, and 30 minutes for the other three tasks (these tasks were a little simpler).

Table 3 shows a summary of the results of a two-way analysis of variance test (with multiple observations per cell) for both the style of interaction and the session duration for the two environments. Since the duration of the sessions were all different, the values of the variables were normalised to a total of 1800 seconds which was the average session duration.

As the results show, neither of the measured variables were affected by the changes made to medium of communication. In fact only one of the ten variables (number of floor changes) gets close to a significant level. This result is entirely consistent with the findings of the earlier study [7], highlighting the fact the style of interaction and the group performance do not seem to be affected by variation in the mode of communication or in the group size.

However, the results of this analysis indicated that there was a difference between the four groups (Table 4) for all the variables measured except the session duration. This finding, which is also consistent with the previous study [7], indicates that the variation among the groups is greater than the variations among the environments. This is due to the fact that different groups are naturally different in terms of the amount of interaction between their members, or the abilities of their members in solving problems. 


\begin{tabular}{lcccc}
\hline & $\begin{array}{c}\text { Face-to-Face } \\
\text { Mean }\end{array}$ & $\begin{array}{c}\text { Voice-Only } \\
\text { Mean }\end{array}$ & $\begin{array}{c}\text { F } \\
\text { df = 1,8 }\end{array}$ & P \\
\hline Maximum Session Duration/Session Duration & 1.45 & 1.42 & 0.01 & 0.912 \\
Speech Duration (s) & 1098 & 1114 & 0.03 & 0.875 \\
Number of Utterances & 380 & 406 & 1.00 & 0.346 \\
Number of Simultaneous Speeches Involving 3 People & 15 & 16 & 0.03 & 0.872 \\
Simultaneous Speech Duration Involving 3 People (s) & 19 & 20 & 0.00 & 0.966 \\
Number of Simultaneous Speeches Involving 2 People & 136 & 133 & 0.02 & 0.894 \\
Simultaneous Speech Duration Involving 2 People (s) & 192 & 176 & 0.32 & 0.585 \\
Mutual Silence Duration (s) & 933 & 901 & 0.28 & 0.609 \\
Number of Floor Changes & 218 & 242 & 4.77 & 0.061 \\
Number of Floor Change Attempts & 78 & 75 & 0.04 & 0.843 \\
\hline
\end{tabular}

Table 3: Summary of two-way ANOVA of interaction style and session duration for media

\begin{tabular}{|c|c|c|c|c|c|c|}
\hline & $\begin{array}{c}\text { Group } 1 \\
\text { Mean }\end{array}$ & $\begin{array}{c}\text { Group } 2 \\
\text { Mean }\end{array}$ & $\begin{array}{c}\text { Group } 3 \\
\text { Mean }\end{array}$ & $\begin{array}{c}\text { Group } 4 \\
\text { Mean }\end{array}$ & $\begin{array}{c}F \\
\mathrm{~d} f=3,8\end{array}$ & $\overline{\mathbf{P}}$ \\
\hline Maximum Session Duration/Session Duration & 1.59 & 1.54 & 1.22 & 1.40 & 0.40 & 0.755 \\
\hline Speech Duration (s) & 716 & 987 & 1839 & 883 & 25.72 & 0.000 \\
\hline Number of Utterances & 253 & 406 & 605 & 309 & 34.48 & 0.000 \\
\hline Number of Simultaneous Speeches Involving 3 People & 8 & 9 & 39 & 7 & 6.65 & 0.014 \\
\hline Simultaneous Speech Duration Involving 3 People (s) & 9 & 11 & 50 & 8 & 5.77 & 0.021 \\
\hline Number of Simultaneous Speeches Involving 2 People & 78 & 102 & 272 & 86 & 23.22 & 0.000 \\
\hline Simultaneous Speech Duration Involving 2 People (s) & 106 & 134 & 377 & 119 & 21.88 & 0.000 \\
\hline Mutual Silence Duration (s) & 1208 & 969 & 439 & 1052 & 31.53 & 0.000 \\
\hline Number of Floor Changes & 144 & 245 & 361 & 170 & 79.24 & 0.000 \\
\hline Number of Floor Change Attempts & 48 & 62 & 152 & 45 & 8.72 & 0.007 \\
\hline
\end{tabular}

Table 4: Summary of two-way ANOVA of interaction style and session duration for groups

\begin{tabular}{lcrrcc}
\hline Question & Size for Test & $\mathbf{W}^{+}$ & $\mathbf{W}^{-}$ & Estimated Median & P \\
\hline $\mathbf{1 . 1}$ & 8 & 23.0 & 13.0 & 0.250 & 0.529 \\
$\mathbf{1 . 2}$ & 9 & 28.5 & 16.5 & 0.500 & 0.515 \\
$\mathbf{1 . 3}$ & 9 & 32.0 & 13.0 & 0.500 & 0.286 \\
$\mathbf{1 . 4}$ & 7 & 21.0 & 7.0 & 0.500 & 0.272 \\
$\mathbf{1 . 5 A}$ & 9 & 41.5 & 3.5 & 1.000 & 0.028 \\
$\mathbf{1 . 5 B}$ & 7 & 25.0 & 3.0 & 1.000 & 0.076 \\
$\mathbf{1 . 5 C}$ & 7 & 22.0 & 6.0 & 0.500 & 0.205 \\
$\mathbf{1 . 5 D}$ & 4 & 7.5 & 2.5 & 0.000 & 0.465 \\
& & & & & \\
$\mathbf{2 . 1}$ & 6 & 11.0 & 10.0 & 0.000 & 1.000 \\
$\mathbf{2 . 2}$ & 3 & 2.0 & 4.0 & 0.000 & 0.789 \\
$\mathbf{2 . 3}$ & 11 & 43.0 & 23.0 & 0.500 & 0.398 \\
$\mathbf{2 . 4}$ & 10 & 18.0 & 37.0 & -0.500 & 0.800 \\
$\mathbf{2 . 5}$ & 7 & 16.0 & 12.0 & 0.000 & 0.673 \\
$\mathbf{2 . 6}$ & 7 & 17.0 & 11.0 & 0.500 & 0.612 \\
$\mathbf{2 . 7}$ & 7 & 17.5 & 10.5 & 0.500 & 1.000 \\
$\mathbf{2 . 8}$ & 1 & 1.0 & 0.0 & 0.000 & 0.834 \\
$\mathbf{2 . 9}$ & 6 & 9.0 & 12.0 & 0.000 &
\end{tabular}

Table 5: Summary of the Wilcoxon Signed Rank Test for questionnaires one and two

\begin{tabular}{lccccc}
\hline Question & Below & Equal & Above & Median & P \\
\hline $\mathbf{3 . 1}$ & 0 & 5 & 7 & 1.000 & 0.016 \\
$\mathbf{3 . 2}$ & 2 & 5 & 5 & 0.000 & 0.453 \\
$\mathbf{3 . 3}$ & 1 & 7 & 4 & 0.000 & 0.375 \\
$\mathbf{3 . 4}$ & 1 & 8 & 3 & 0.000 & 0.625 \\
$\mathbf{3 . 5}$ & 3 & 2 & 7 & 1.000 & 0.344 \\
\hline
\end{tabular}

Table 6: Summary of the Sign Test for questionnaire three 


\subsection{Questionnaires}

The analysis of the questionnaires was reasonably simple. The subjects' answers to the questions were truncated to the nearest whole number. For questionnaires one and two (expectations and reactions) the Wilcoxon signed rank test [11] was used to compare the values subjects gave to different questions in the two environments. The result of this analysis is shown in Table 5.

The $\mathrm{W}^{+}$and $\mathrm{W}^{-}$values in Table 5 show the sum of the ranks with a positive or negative sign respectively. The Size for Test value on the other hand shows the number of answers which were different for the two environments (note that there were 12 pairs of answers for each question).

As the result show, the only question for which the subjects' answers for the two environments were different to a significant level was question $1.5 \mathrm{~A}$. This means that the subjects expected that the audio-based communication mode would be less friendlier than the face-to-face mode. Once again this finding is completely consistent with the result of the earlier study [5], indicating that increasing the group size from two to three had no real effect in the subjects' expectations or reactions to the audio-based and face-to-face communication modes.

Questionnaire three, which was used for obtaining the subjects' ranking of the two environments, was analysed in a slightly different manner using the sign test [11]. The result of this test which is shown in Table 6 indicates that only for question 3.1 did the subjects rank the face-to-face environment differently to the audio-based environment. This means that the subjects felt that working in the faceto-face mode was more enjoyable than working in the voice-only mode. Note that the values for Below, Equal, and Above show the number of people who felt that the face-to-face mode was worse, the same, or better than the voice-only environment respectively.

Although the result of this questionnaire is different from the result of a similar questionnaire used in the previous experiment [5], it is reasonable to say that

\section{References}

1. Argyle, M. and Cook, M., Gaze and Mutual Gaze. Cambridge University Press, 1976.

2. Chapanis, A., Interactive Human Communication. Scientific American, 232, (3), 1975, 36-42.

3. Gale, S., Human Aspects of Interactive Multimedia Communication. Interacting with Computers, 2, (2), 1990, 175-189.

4. Group Technologies, Inc., Aspects: The first Simultaneous Conference Software for the Macintosh, 1990.

5. Masoodian, M. and Apperley, M., User Perceptions of Human-to-Human Communication Modes in CSCW Environments. ED-MEDIA '95, World Conference on Educational Multimedia and Hypermedia, 1995, 430-435. because there were fewer environments in the current experiment the subjects were able to make a better comparison of them. It should also be noted that even though the face-to-face and voice-only environments were ranked differently in the previous experiment, the subjects indicated that there was very little difference between them.

\section{Conclusions}

The results of the data analysis discussed in this paper along with the results of the previous work [5, 6, 7], indicate that an audio channel is an important and sufficient means of communication for supporting sharedworkspace collaboration in a problem-solving situation.

Furthermore, these results also show that there is very little change, if any, produced by shifting from audio-only to a face-to-face situation, apart from the more "natural" feeling some participants felt in the latter environment. On a communication bandwidth scale, video-based communication would come somewhere between these two $[5,6,7]$ so it can be concluded that there would be no significant advantage of video over audio with a sharedworkspace problem-solving task.

What is even more interesting is that, for this type of task, changing the group size from two to three seems to have no effect on the measured factors. It should however be noted that since groups of size four or more are once again different from groups of size three [13], similar studies with larger groups may give different results.

In summary, the current series of empirical studies reported here show that for small collaborative problemsolving groups an audio channel plus a computer supported shared-workspace is sufficient for satisfactory and productive group work. Therefore, rather than attempting to integrate video and other means of communication with audio to support remote sharedworkspace collaboration, industry should be devoting more attention to improving groupware technology which is used to provide the shared-workspace facility for remote collaborative work.

6. Masoodian, M., Apperley, M., and Frederikson, L., The Impact of Human-to-Human Communication Modes in CSCW Environments. Proceedings of OZCHI'94, the CHISIG Annual Conference on Human-Computer Interaction, 1994, 193-198.

7. Masoodian, M., Apperley, M., and Frederikson, L., Video Support for Shared Work-Space Interaction - An Empirical Study. Interacting with Computers, 7, (3), 1995, 237-253.

8. McCarthy, J. C. and Monk, A. F., Measuring the Quality of Computer-Mediated Communication. Behaviour and Information Technology, 13, (5), 1994, 311-319.

9. McGrath, J. E., Groups: Interaction and Performance. Prentice-Hall, 1984. 
10. Minneman, S. L. and Bly, S. A., Managing a trois: A Study of a Multi-User Drawing Tool in Distributed Design Work. Proceedings of ACM CHI'91 Conference on Human Factors in Computing Systems, 1991, 217-224.

11. Neter, J., Wasserman, W., and Whitmore, G. A., Applied Statistics. Third Edition, Allyn and Bacon, 1988.

12. Ochsman, R. B. and Chapanis, A., The Effects of 10 Communication Modes on the Behavior of Teams During Co-operative Problem-Solving. International Journal of Man-Machine Studies, 6, (5), 1974, 579-619.

13. Olson, J. S., Olson, G. M., and Meader, D. K., What Mix of Video and Audio Is Useful for Small Groups Doing Remote Real-Time Design Work? Proceedings of ACM CHI'95, Conference on Human Factors in Computing Systems, 1995, 362-368.

14. Rutter, D. R. and Stephenson, G. M., The Role of Visual Communication in Synchronising Conversation. European Journal of Social Psychology, 7, (1), 1977, 29-37.

15. Rutter, D. R., Stephenson, G. M., and Dewey, M. E., Visual Communication and the Content and Style of Conversation. British Journal of Social Psychology, 20, 1981, 41-52.

16. Sellen, A. J., Remote Conversations: The Effects of Mediating Talk with Technology. Human-Computer Interaction, 10, (4), 1995, 401-437.
17. Short, J., Williams, E., and Christie, B., The Social Psychology of Telecommunication. John Wiley \& Sons, 1976.

18. Smith, R. B., O'Shea, T., O'Malley, C., Scanlon, E., and Taylor, J., Preliminary Experiences with a Distributed, Multi-media, Problem Solving Environment. In Bowers, J. M. and Benford, S. D. (Eds), Studies in Computer Supported Cooperative Work: Theory, Practice and Design. Elsevier Science Publishers, 1991, 31-48.

19. Tang, J. C., and Isaacs, E., Why Do Users Like Video? Studies of Multimedia-Supported Collaboration. Computer Supported Cooperative Work An International Journal (CSCW), 1, (3), 1993, 163-196.

20. Valacich, J. S., Dennis, A. R., and Connolly, T., Idea Generation in Computer-Based Groups: A New Ending to an Old Story. Organizational Behavior and Human Decision Processes, 57, 1994, 448-467.

21. Whittaker, S., Geelhoed, E., and Robinson, E., Shared Workspaces: How Do They Work and When Are They Useful? International Journal of Man-Machine Studies, 39, (5), 1993, 813-842.

22. Williams, E., Experimental Comparison of Face-to-Face and Mediated Communication: A Review. Psychological Bulletin, 84, (5), 1977, 963-976. 